\title{
Supporting Customer Choice with Semantic Similarity Search and Explanation*
}

\author{
Anna Formica, Michele Missikoff, Elaheh Pourabbas, and Francesco Taglino \\ National Research Council, Istituto di Analisi dei Sistemi ed Informatica "A. Ruberti" \\ Viale Manzoni 30, I-00185 Rome, Italy \\ \{name.surname\} @iasi.cnr.it
}

\begin{abstract}
Semantic search and retrieval methods have a great potentiality in helping customers to make choices, since they appear to outperform traditional keyword-based approaches. In this paper, we address SemSim, a semantic search method based on the well-known information content approach. SemSim has been experimented to be effective in a defined domain, namely the tourism sector. During experimentation, one of the first requests raised from the users concerned the possibility to explain, besides the typical output of a semantic search engine, why a given result was returned. In this paper we investigate SemSim with the aim of providing the user with an explanation about the motivations behind the ranked list of returned options, with graphical representations conceived to better visualize the results of the semantic search.
\end{abstract}

Keywords: Similarity Reasoning, Weighted Reference Ontology, Information content, Digital Resources.

\section{Introduction}

Similarity reasoning represents a promising application of semantic technologies that is constantly gaining importance in information management. It also represents one of the key pillars of a new generation of search engines, capable of processing a user request from a conceptual perspective instead of basing the research on keyword-matching mechanisms. Along this direction, several semantic approaches have been proposed in the literature, based on the use of reference ontologies. Such approaches require that both the user request and the elements of the search space are semantically annotated, i.e., are associated with semantic expressions built upon the concepts of a reference ontology. This approach, referred to as semantic search and retrieval, appears to outperform traditional keyword-based approaches. However, the application of semantic search is restricted to focused domains due to the cost needed to develop the required semantic infrastructures (basically, reference ontology and semantic annotations).

In a previous paper [5] the authors have proposed SemSim, a semantic search method based on the idea that the similarity between conceptual structures depends on the amount of information content they share. In turn, the information content carried by a

\footnotetext{
* This work has been partially supported by the European project BIVEE: Business Innovation and Virtual Enterprise Environment (No. FoF-ICT-2011.7.3-285746).
} 
conceptual structure depends on the information content carried by the concepts (features) of the reference ontology (an ontology is a formal, explicit specification of a shared conceptualization [7]) for the given domain, at a given time. Intuitively, the information content of a concept, in our approach, depends on the number of objects in the search space that carry such a concept. In brief, a concept associated with fewer objects has a better selection coefficient and therefore a greater information content. Conversely, a concept that can be found in a large number of objects has less selectivity and therefore carries a more limited information content. Then, we introduced the notion of a weighted ontology, where each concept is associated with a weight corresponding to the frequency of the search objects that carry such a concept. It is quite obvious that concepts up in the hierarchy will have higher weight (and therefore less information content) than more specific concepts, positioned down in the hierarchy [11].

The core of the SemSim method consists in the derivation of the semantic similarity between concept structures, one representing a user request and one representing a target resource. Currently, we are experimenting the method, and the possibility to obtain, besides the typical output of a semantic search engine (i.e., a list of target objects, ranked according to a similarity score), a synthetic explanation of why the similarity engine achieves the displayed results. Therefore, the objective of this work is to extend the SemSim method by providing the user with such an explanation about the mechanism adopted in deriving the ranked list of objects. It is worth noting that often the outcome is not intuitive, since SemSim aims at maximizing the global information sharing of the user request and target object (i.e., the summation of the common information contents for all the concepts characterizing a given search object) therefore, avoiding 'easy' matching mechanisms.

The paper is organized as follows. After the next section presenting the related work, Section 3 recalls some basic notions of the SemSim method. In Section 4, a qualitative analysis of SemSim is presented, and in Section 5 two different visualizations of the analyzed results are given. Finally, Section 6 concludes.

\section{Related Work}

In the large majority of papers proposed in the literature [3,13], semantic search is performed by using WordNet [16], see for instance [8,15]. WordNet (a lexical ontology for the English language) provides, for a given concept (noun), the natural language definition, hypernyms, hyponyms, synonyms, etc, and also a measure of the frequency of the concept, by using noun frequencies from the Brown Corpus of American English [6]. We did not adopt the WordNet frequencies for two reasons. Firstly, we deal with specialized domains (e.g., systems engineering, tourism, etc.), requiring specialized domain ontologies. WordNet is a generic lexical ontology (i.e., not focused on a specific domain) that contains only simple terms. In fact, multi-word terms are not reported (e.g., terms such as "private house" or "country resort" are not defined in WordNet). Secondly, there are concepts in WordNet for which the frequency is not given (e.g., "accommodation").

The work presented in [8] shares some analogies with our approach with regard to the need of computing weights without relying on large text corpora. Therefore, they propose a method, referred to as $\mathrm{CP} / \mathrm{CV}$, such that each node in the taxonomy is 
associated with a concept vector, built on the basis of the topology of the ontology and the position of concepts therein. Then, the similarity of concepts is evaluated according to the cosine similarity of the related concept vectors. Conversely, in our work the similarity of concepts is conceived to determine the similarity of two concept vectors.

Regarding the methods to compute the similarity between concept vectors, our work proposes a two stages method, firstly computing the pair-wise concept similarity (consim), and then deriving the similarity between vectors of concepts (semsim). Pairwise concept similarity is performed according to the information content approach [11]. With regard to the second stage, we adopted a solution inspired by the maximum weighted matching problem in bipartite graphs. In the literature the Dice, Jaccard and Cosine [12] methods are often adopted in order to compare vectors of concepts. However, in these methods the matchmaking of two concept vectors is based on their intersection, without considering the position of the concepts in the ontology. According to the Weighted Sum approach [2] a fixed value (i.e., 0.5) is assigned to each pair of hierarchically related concepts. Our proposal is based on a more refined semantic matchmaking, since the match of two concepts is performed according to their shared information content, and the vector similarity is based on the optimal concept coupling.

Within the semantic web search approaches supported by ontologies, we selected [1] and [10] as representative works. In the mentioned papers, semantic search methods have been conceived for retrieving the relevant resources based on $\mathrm{OWL}^{1}$ (Ontology Web Language) ontologies. Basically, the relevant resources are identified by weighting the instances and properties defined in the assertional knowledge (ABox). Conversely, in our approach, weights are assigned to the features which correspond to the concepts of the terminological knowledge (TBox), and the identification of the relevant resources is performed on the basis of the ranked feature vectors.

\section{The Semsim Method: An Overview}

In [5] two different SemSim methods were addressed, namely the probabilistic and the frequency approaches. In the mentioned paper, we have shown that the SemSim method based on the frequency approach has a higher correlation with Human Judgement $(H J)$ than some of the most representative approaches defined in the literature. In this section, we recall the frequency approach, which is the focus of our paper. The Universe of Digital Resources (UDR) is the totality of digital resources that are semantically annotated with a reference ontology. In our work we address a simplified notion of ontology, Ont, consisting of a set of concepts organized according to a ISA hierarchy. In particular, Ont is a taxonomy defined by the pair $O n t=\langle C, H>$, where $C$ is a set of concepts and $H$ is a set of ordered pairs of concepts of $C$ such that if $\left(c_{i}, c_{j}\right)$ $\in H$, then $c_{j}$ ISA $c_{i}$, i.e., $c_{i}$ is a more general concept with respect to $c_{j}$.

Given two concepts $c_{i}, c_{j} \in C$, the least upper bound of $c_{i}, c_{j}, \operatorname{lu} b\left(c_{i}, c_{j}\right)$, is always uniquely defined in $C$ (we assume the hierarchy is a lattice). It represents the least abstract concept of the ontology that is a more general with respect to $c_{i}$ and $c_{j}$.

\footnotetext{
${ }^{1}$ http: / / www .w3 . org/TR/ow12-primer/
} 
Consider an ontology Ont $=\langle C, H\rangle$. A request feature vector (request vector for short) $r v$ is defined by a set of ontology concepts (the order of the concepts is irrelevant), i.e., $r v=\left(c_{1}, \ldots, c_{n}\right)$ where $c_{i} \in C$.

Analogously, given a digital resource $d r_{i} \in \mathrm{UDR}$, an ontology feature vector $o f v_{i}$ associated with $d r_{i}$ is defined by a set of ontology concepts describing the resource as follows: of $v_{i}=\left(c_{i, 1}, \ldots, c_{i, m}\right)$, where $c_{i, j} \in C, j=1, \ldots, m$.

A Weighted Reference Ontology $(W R O)$ is a pair $W R O=\langle O n t, w\rangle$, where $w$ is a function defined on $C$, such that given a concept $c \in C, w(c)$ is a rational number in the interval $[0, \ldots, 1]$.

In the following, let $H^{*}$ be the reflexive and transitive closure of $H$. According to the frequency approach, the weight associated with the concept $c$ in the ontology Ont $=<C, H>$, indicated as $w_{f}(c)$, is defined as follows:

$$
w_{f}(c)=\frac{\left|\bigcup\left\{o f v:\left(c^{\prime} \in o f v,\left(c, c^{\prime}\right) \in H^{*}\right)\right\}\right|}{|U D R|}
$$

Therefore, the weight assignment is computed on the basis of the given ontology feature vectors and weights are assigned according to a bottom-up approach in order to take into account the essence of the ISA hierarchy. For instance, in our running example in the tourism domain, suppose the digital resources are vacation packages for visiting a European capital, which are offered by a tourism agency. Each package is annotated with one ontology feature vector defined by using the concepts of the WRO shown in Figure 1. Suppose we have a UDR containing 22 vacation packages, each annotated as shown in Table 1.

Table 1. Annotation of vacation packages based on ontology feature vector

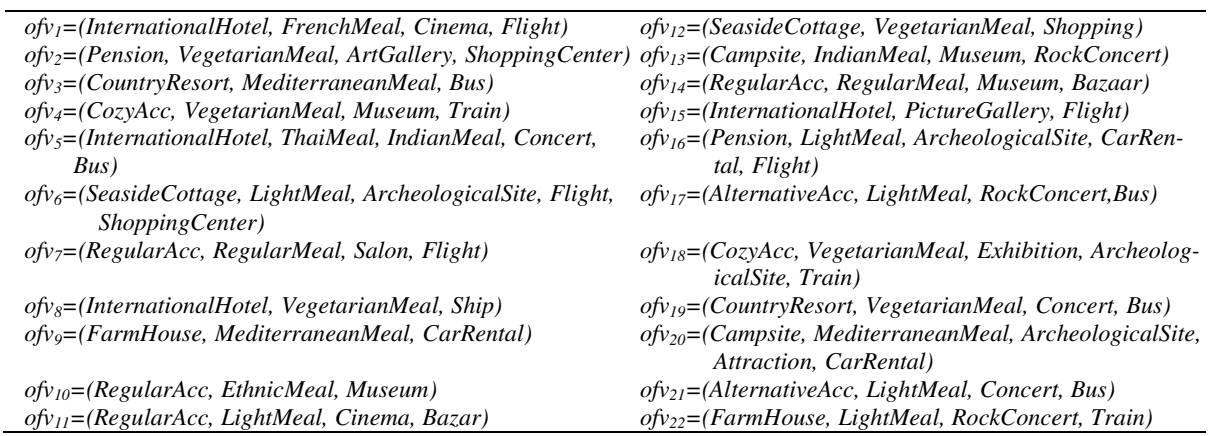

Consider the feature VegetarianMeal (a leaf in the taxonomy tree) which appears in each of the six ofvs of the set $\left\{o f v_{2}, o f v_{4}, o f v_{8}, o f v_{12}, o f v_{18}, o f v_{19}\right\}$ (see Table 1). The relative frequency of this feature over the whole resources (the 22 packages) is $w_{f}($ VegetarianMeal $)=6 / 22=0.27($ see Figure 1$)$. 


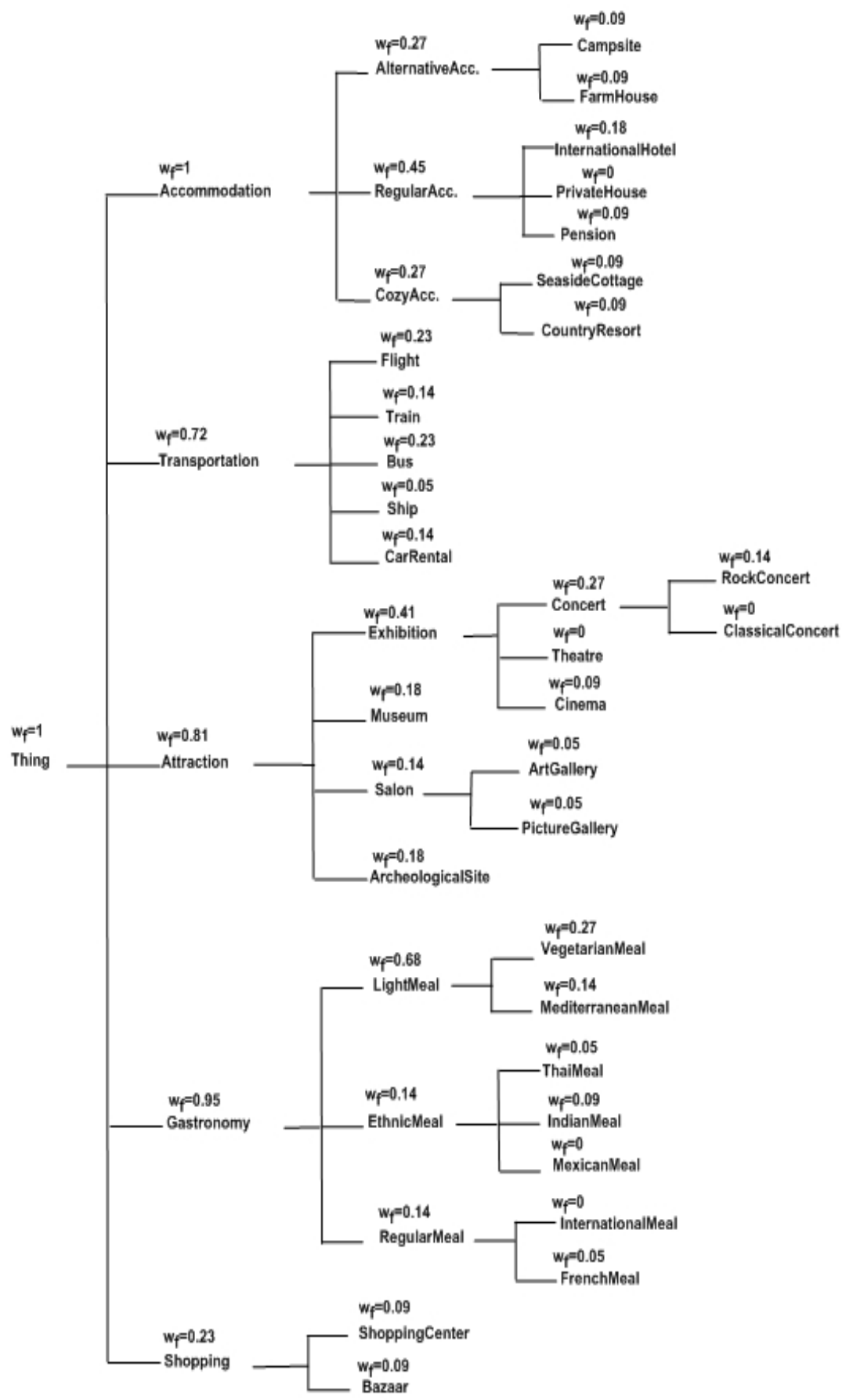

Fig. 1. WRO of our running example 
In order to assign a weight to a more general concept, we have to consider not only the set of ofvs containing the concept but also the sets of ofvs containing its descendants in the ISA hierarchy. For instance, consider LightMeal which has only two children as descendants, namely VegetarianMeal and MediterraneanMeal. MediterraneanMeal appears in the ofvs of the set $\left\{o f v_{3}\right.$, of $v_{9}$, of $\left.v_{20}\right\}$. LightMeal is contained in the ofvs of the set $\left\{o f v_{6}, o f v_{11}, o f v_{16}, o f v_{17}, o f v_{21}, o f v_{22}\right\}$, therefore its weight is given by the cardinality of the union of the three sets above, divided by the total number of ofvs, i.e., $w_{f}($ LightMeal $)=15 / 22=0.68$. Note that, in the case of overlapping sets of ofvs, the union set in the above formula guarantees that $\mathrm{w}_{\mathrm{f}}$ does not exceed one.

Let us consider a tourist who wants to visit a European capital and, in order to buy a vacation package, he/she expresses the following preferences:

"I would like travel by bus, sleep in a campsite, have ethnic food, and enjoy rock concerts".

It can be formulated according to the request feature vector notation as follows:

$$
r v_{1}=(\text { Campsite, EthnicMeal, RockConcert, Bus). }
$$

The SemSim method allows the user to choose among the vacation packages offered by the tourism agency of our running example (shown in Table 1) the one that better satisfies his/her needs. In particular, it evaluates the similarity between feature vectors (semsim), which is based on the notion of similarity between concepts (features), referred to as consim.

Given a WRO, the consim method relies on the information content approach defined by Lin [11]. According to the standard argumentation of information theory, the information content of a concept $c$ is defined as $-\log w(c)$, where $w$ is the weight associated with the concept $c$ in the WRO. Therefore, as the weight of a concept increases the informativeness decreases hence, the more abstract a concept the lower its information content. Given two concepts $c_{i}$ and $c_{j}$, their similarity, $\operatorname{consim}\left(c_{i}, c_{j}\right)$, is defined as the maximum information content shared by the concepts divided by the sum of the information contents of the concepts. Note that we assume the ontology is a tree, therefore the least upper bound of $c_{i}$ and $c_{j}, \operatorname{lub}\left(\mathrm{c}_{i}, \mathrm{c}_{j}\right)$, is always defined and provides the maximum information content shared by the concepts in the taxonomy. Formally, we have:

$$
\operatorname{consim}\left(c_{i}, c_{j}\right)=2 \times \frac{\log w_{f}\left(\operatorname{lub}\left(c_{i}, c_{j}\right)\right)}{\log w_{f}\left(c_{i}\right)+\log w_{f}\left(c_{j}\right)}
$$

For instance, consider the pair of concepts VegetarianMeal and MediterraneanMeal of the WRO shown in Figure 1. The consim measure between VegetarianMeal and MediterraneanMeal is defined as follows:

$$
\begin{gathered}
\operatorname{consim}(\text { VegetarianMeal,MediterraneanMeal })=2 \log w_{f}(\text { LightMeal }) /\left(\log w_{f}\right. \\
\left.(\text { VegetarianMeal })+\log w_{f}(\text { MediterraneanMeal })\right)=0.24 .
\end{gathered}
$$

In fact, LightMeal is the lub between VegetarianMeal and MediterraneanMeal, and therefore provides the maximum information content shared by the comparing concepts. 
As anticipated, the SemSim method allows us to compute the semantic similarity between the request vector $r v$ and an $o f v$, $\operatorname{sem} \operatorname{sim}(r v, o f v)$, by using the consim similarity above. We start by computing the consim for each pair of concepts of the Cartesian product of the two vectors. However, we are not interested in all possible pairs because in many cases the comparison is meaningless (e.g., contrasting VegetarianMeal with Concert). Hence, we restrict our analysis to the pairs that exhibit high affinity. In particular, we consider the stable marriage problem [14], which is the problem of finding a stable matching (i.e., there does not exist any better alternative pairing) between sets of elements. For instance, assuming $r v$ and $o f v$ represent a set of boys and a set of girls respectively, we analyze all possible sets of marriages, when polygamy is not allowed. Our solution, for the computation of the semantic similarity makes use of the Hungarian algorithm as an implementation for solving the maximum weighted matching problem in bipartite graphs [9] which runs in polynomial time. Essentially, the method aims to identify the set of pairs of concepts of the two vectors that maximizes the sum of the consim similarity values:

$$
\operatorname{semsim}(r v, o f v)=\frac{\max \left(\sum \operatorname{consim}\left(c_{i}, c_{j}\right)\right)}{\max (n, m)}
$$

where: $i=1 . . n, j=1 . . m, n=|r v|, m=|o f v|, c_{i} \in r v$, and $c_{j} \in o f v$.

For instance, in the case of the request vector $r v_{1}$ defined above and the $o f v_{21}$ shown in Table 1, the following set of pairs of concepts (enclosed in parenthesis) has the maximum consim sum, as shown below:

$$
\begin{aligned}
& \text { consim }(\text { Campsite, AlternativeAcc. })=0.70 \\
& \text { consim }(\text { EthnicMeal, LightMeal })=0.04 \\
& \text { consim }(\text { RockConcert, Concert })=0.79 \\
& \text { consim }(\text { Bus, Bus })=1
\end{aligned}
$$

Therefore: $\operatorname{semsim}\left(r v_{1}, o f v_{21}\right)=(0.70+0.04+0.79+1) / 4=0.63$, where the sum of consim has been normalized according to the maximum cardinality of the contrasted vectors (in this case 4).

\section{Semsim Experimental Analysis}

In [5] we have presented an experiment where SemSim has been compared to some of the most representative similarity methods defined in the literature. In the experiment four request vectors have been defined, $r v_{i}, i=1, \ldots, 4$, corresponding to the vacation preferences of four different tourists, which are recalled in Table 2. The correlation between each selected similarity method and human judgment $(H J)$ has been evaluated, where $H J$ values had been obtained by asking to a group of 21 people, randomly selected from our Institute staff, to indicate the similarity among each $r v_{i}$ and the vacation packages $o f v_{\mathrm{i}}, \mathrm{j}=1, \ldots, 22$, defined in Table 1 . As already mentioned, in [5] our experiment has shown that the SemSim method based on the frequency approach has a higher correlation with $H J$ than the other approaches selected in the literature. Therefore, in this section, our analysis is focused on the SemSim method based on the frequency approach. 
In Table 3, the SemSim values obtained according to our experiment are recalled. The ofvs are listed with the associated similarity values, starting from the highest up to the lowest values.

Table 2. Request Vectors

\begin{tabular}{l}
\hline $\mathrm{rv}_{1}=$ (Campsite, EthnicMeal, RockConcert, Bus) \\
$\mathrm{rv}_{2}=$ (InternationalHotel, InternationalMeal, ArtGallery, Flight $)$ \\
$\mathrm{rv}_{3}=$ (Pension, MediterraneanMeal, Cinema, ShoppingCenter $)$ \\
$\mathrm{rv}_{4}=$ (CountryResort, LightMeal, ArcheologicalSite, Museum, Train) \\
\hline
\end{tabular}

Let us consider the first five ofvs listed in Table 3, i.e., the ofvs associated with the five highest SemSim values. For each $r v_{i} i=1, \ldots, 4$, a table has been defined, namely Tables 4, 5, 6, and 7 respectively, where the ofus with the five highest values have been indicated. In each table, in the first row the features of the addressed request vector are listed, and in the remaining rows, the features of the selected ofvs are recalled. The positioning of the features of the ofvs in the rows has been performed in order to show the set of pairs of features (one belonging to the request vector and the other one to the ofv) leading to the maximum weighted matching value, according to the stable marriage problem recalled above. In the rows, the features are shown with the related consim values (in square brackets) and a label (one among $\mathrm{M}, \mathrm{H}, \mathrm{S}, \mathrm{C}$ and R) specifying the kind of relation existing between the matched features, as explained below:

- M stands for exact match, therefore the consim value is 1.0;

- H stands for hierarchical relation, i.e. one feature is a descendant/ancestor of the other; in parenthesis the number of arcs connecting the two features is also given (e.g., H(1) stands for a parent/child relation);

- S stands for sibling, i.e., children of the same father;

- C stands for cousin, i.e., children of siblings;

- R stands for related, for all the remaining pairs of features of the ontology.

Let us consider for instance Table 4, related to the request vector $r v_{l}$. According to the maximum weighted matching problem, the ofv with the highest SemSim value with $r v_{1}$ is $o f v_{13}$, which has four features. The pairs of features providing this value are (Campsite, Campsite), (EthnicMeal, IndianMeal), (RockConcert, RockConcert), and (Bus, Museum). Therefore, two features, namely Campsite and RockConcert, match exactly (M), IndianMeal is in the hierarchical relation $\mathrm{H}(1)$ with EthnicMeal, because the former is a child of the latter, and the other feature, Museum, is related to Bus according to the cousin relation (C). In square brackets we represent the consim values associated with these pairs, which in the case of the pair (Bus, Museum) is zero because these features do not share any information in the ontology except for Thing. In analyzing the four tables, as a general observation, note that the ofvs with higher SemSim values show a greater number of pairs of features with exact matches (M) with respect to the other ofvs. Immediately lower SemSim values correspond to a less number of exact matches $M$ and a high number of parent/child relations $\mathrm{H}(1)$. Going down in the tables, the lowest SemSim values are associated with a higher number of related features $(\mathrm{R})$, because $\mathrm{R}$ does not represent any specific relation between the features in the ontology. For instance, in the case of the request vector $r v_{2}$, in the first 
row of Table 5 we have the $o f v_{15}$ showing two exact matches and one sibling relation, and in the second row we find the $o f v_{7}$, for which one single match and three parent/child relations are present.

Table 3. Ranking results for request vectors

\begin{tabular}{|c|c|c|c|c|c|c|c|}
\hline \multicolumn{2}{|c|}{$r v_{1}$} & \multicolumn{2}{|c|}{$r v_{2}$} & \multicolumn{2}{|c|}{$r v_{3}$} & \multicolumn{2}{|c|}{$r v_{4}$} \\
\hline $\begin{array}{c}\text { Ranked } \\
\text { Resources }\end{array}$ & Value & $\begin{array}{c}\text { Ranked } \\
\text { Resources }\end{array}$ & Value & $\begin{array}{c}\text { Ranked } \\
\text { Resources }\end{array}$ & Value & $\begin{array}{c}\text { Ranked } \\
\text { Resources }\end{array}$ & Value \\
\hline$o f v_{13}$ & 0.73 & $o f v_{15}$ & 0.66 & $o f v_{11}$ & 0.61 & $o f v_{18}$ & 0.66 \\
\hline of $v_{17}$ & 0.69 & of $v_{7}$ & 0.60 & of $v_{2}$ & 0.58 & ofv $_{4}$ & 0.63 \\
\hline of $v_{21}$ & 0.63 & $o f v_{1}$ & 0.52 & $o f v_{1}$ & 0.35 & of $v_{6}$ & 0.55 \\
\hline of $v_{5}$ & 0.54 & $o f v_{2}$ & 0.35 & of $v_{14}$ & 0.31 & $o f v_{16}$ & 0.44 \\
\hline of $v_{19}$ & 0.45 & $o f v_{16}$ & 0.29 & $o f v_{6}, o f v_{16}$ & 0.28 & $o f v_{22}$ & 0.42 \\
\hline$o f v_{22}$ & 0.44 & $o f v_{8}$ & 0.28 & $\begin{array}{c}o f v_{3}, o f v_{9} \\
o f v_{12}\end{array}$ & 0.25 & $o f v_{19}$ & 0.35 \\
\hline of $v_{10}$ of $v_{20}$ & 0.28 & $o f v_{5}$ & 0.26 & of $v_{20}$ & 0.23 & $o f v_{20}$ & 0.34 \\
\hline of $v_{3}$ & 0.26 & of $v_{6}$ & 0.22 & of $v_{21}$ & 0.20 & ofv 3 & 0.30 \\
\hline$o f v_{9}$ & 0.19 & $\begin{array}{c}\text { of } v_{10}, \text { of } v_{11}, \\
\text { of } v_{14}\end{array}$ & 0.18 & $o f v_{5}, o f v_{17}$ & 0.18 & $o f v_{17}, o f v_{21}$ & 0.26 \\
\hline$o f v_{18}$ & 0.17 & $o f v_{19}, o f v_{21}$ & 0.08 & $o f v_{19}, o f v_{22}$ & & $o f v_{13}$ & 0.23 \\
\hline$o f v_{I}$ & 0.16 & $\begin{array}{c}o f v_{4}, o f v_{17} \\
o f v_{22}\end{array}$ & 0.07 & $o f v_{18}$ & 0.16 & $o f v_{11}$ & 0.22 \\
\hline$o f v_{I I}$ & 0.11 & $o f v_{18}, o f v_{20}$ & 0.06 & $\begin{array}{c}o f v_{7}, \text { of } v_{8} \\
o f v_{10}\end{array}$ & 0.15 & $o f v_{10}, o f v_{14}$ & 0.21 \\
\hline$o f v_{4}, o f v_{7}$ & 0.08 & $o f v_{3}, o f v_{9}$ & 0.05 & $o f v_{13}, o f v_{15}$ & 0.11 & $o f v_{12}$ & 0.20 \\
\hline $\begin{array}{l}\text { of } v_{6,} \text { of } v_{15} \\
\text { of } v_{16}\end{array}$ & 0.07 & $o f v_{13}$ & 0.02 & $o f v_{4}$ & 0.08 & $o f v_{8}$ & 0.12 \\
\hline ofv 8 & 0.04 & $o f v_{12}$ & 0.00 & & & $o f v_{2}$ & 0.11 \\
\hline$o f v_{2}, o f v_{14}$ & 0.03 & & & & & of $v_{9}$ & 0.10 \\
\hline of $v_{16}$ & 0.01 & & & & & $\begin{array}{c}o f v_{5}, o f v_{7} \\
o f v_{1}\end{array}$ & $\begin{array}{l}0.07 \\
0.06\end{array}$ \\
\hline & & & & & & $o f v_{15}$ & 0.05 \\
\hline
\end{tabular}

However, this does not hold in general. In fact in the same table, of $v_{l}$ in the third position has one more exact match with respect to $o f v_{7}$. The reason is that, in the case of $o f v_{1}$, two exact matches are combined with a cousin relation which has a very low consim value. We have a similar situation in Table 6, for the $o f v_{11}$ and $o f v_{2}$ in the case of the request vector $r v_{3}$. In fact, the combination of one exact match (Cinema) with two parent/child relations (Pension, RegularAcc) and (MediterraneanMeal, LightMeal) leads to a higher SemSim value for the of $v_{11}$ with respect to the of $v_{2}$ for which two exact matches (Pension) and (ShoppingCenter) are combined with a sibling relation (MediterraneanMeal, VegetarianMeal) and a cousin relation (Cinema, ArtGallery), the last one with a very low value.

Table 4. Ranking results for $r v_{1}$

\begin{tabular}{|c|c|c|c|c|c|c|}
\hline$r v_{1}$ & Campsite & EthnicMeal & RockConcert & Bus & & Semsim \\
\hline$o f v_{13}$ & $\begin{array}{l}\text { Campsite } \\
\text { M [1.0] }\end{array}$ & $\begin{array}{l}\text { IndianMeal } \\
\mathrm{H}(1)[0.91]\end{array}$ & $\begin{array}{c}\text { RockConcert } \\
\text { M [1.0] }\end{array}$ & $\begin{array}{c}\text { Museum } \\
\text { C [0] }\end{array}$ & & 0.73 \\
\hline$o f v_{17}$ & $\begin{array}{c}\text { AlternativeAcc } \\
\mathrm{H}(1)[0.70]\end{array}$ & $\begin{array}{c}\text { LightMeal } \\
\mathrm{S}[0.04]\end{array}$ & $\begin{array}{c}\text { RockConcert } \\
\text { M [1.0] }\end{array}$ & $\begin{array}{c}\text { Bus } \\
\text { M [1.0] }\end{array}$ & & 0.69 \\
\hline$o f v_{21}$ & $\begin{array}{c}\text { AlternativeAcc } \\
\mathrm{H}(1)[0.70]\end{array}$ & $\begin{array}{c}\text { LightMeal } \\
\mathrm{S}[0.04] \\
\end{array}$ & $\begin{array}{c}\text { Concert } \\
\mathrm{H}(1)[0.79] \\
\end{array}$ & $\begin{array}{c}\text { Bus } \\
\text { M [1.0] }\end{array}$ & & 0.63 \\
\hline$o f v_{5}$ & $\begin{array}{c}\text { InternationalHotel } \\
\mathrm{C}[0] \\
\end{array}$ & $\begin{array}{l}\text { IndianMeal } \\
\mathrm{H}(1)[0.91]\end{array}$ & $\begin{array}{c}\text { Concert } \\
\mathrm{H}(1)[0.79] \\
\end{array}$ & $\begin{array}{c}\text { Bus } \\
\text { M [1.0] }\end{array}$ & $\begin{array}{c}\text { ThaiMeal } \\
{[0]}\end{array}$ & 0.54 \\
\hline$o f v_{19}$ & $\begin{array}{c}\text { CountryResort } \\
\text { C }[0]\end{array}$ & $\begin{array}{l}\text { VegetarianMeal } \\
\text { R [0.03] }\end{array}$ & $\begin{array}{c}\text { Concert } \\
H(1)[0.79]\end{array}$ & $\begin{array}{c}\text { Bus } \\
\text { M [1.0] }\end{array}$ & & 0.45 \\
\hline
\end{tabular}


Consider now the of $v_{5}$ in Table 4. It contains one more feature than the request vector $r v_{l}$ and, according to the stable marriage problem, it will necessarily have a dangling feature in the table. This feature is ThaiMeal, even though in the request vector there is EthnicMeal which is its parent. This is due to the higher consim similarity value between IndianMeal and EthnicMeal (0.91) than ThaiMeal and EthnicMeal (0.78). For this reason, since ThaiMeal does not contribute in any way to SemSim value, the value in square brackets has been set to zero.

Table 5. Ranking results for $r v_{2}$

\begin{tabular}{|c|c|c|c|c|c|}
\hline$r v_{2}$ & InternationalHotel & InternationalMeal & ArtGallery & Flight & Semsim \\
\hline$o f v_{15}$ & $\begin{array}{c}\text { InternationalHotel } \\
\mathrm{M}[1.0]\end{array}$ & & $\begin{array}{c}\text { PictureGallery } \\
\text { S [0.64] }\end{array}$ & $\begin{array}{l}\text { Flight } \\
\text { M [1.0] }\end{array}$ & 0.66 \\
\hline$o f v_{7}$ & $\begin{array}{l}\text { RegularAcc } \\
\mathrm{H}(1)[0.63] \\
\end{array}$ & $\begin{array}{c}\text { RegularMeal } \\
\text { H(1) [0] }\end{array}$ & $\begin{array}{c}\text { Salon } \\
\mathrm{H}(1)[0.78]\end{array}$ & $\begin{array}{l}\text { Flight } \\
\text { M [1.0] } \\
\end{array}$ & 0.60 \\
\hline$o f v_{1}$ & $\begin{array}{c}\text { InternationalHotel } \\
\mathrm{M}[1.0]\end{array}$ & $\begin{array}{c}\text { FrenchMeal } \\
\text { S [0] }\end{array}$ & $\begin{array}{l}\text { Cinema } \\
\mathrm{C}[0.07]\end{array}$ & $\begin{array}{c}\text { Flight } \\
\text { M [1.0] }\end{array}$ & 0.52 \\
\hline$o f v_{2}$ & $\begin{array}{l}\text { Pension } \\
\mathrm{S}[0.38]\end{array}$ & $\begin{array}{c}\text { VegetarianMeal } \\
\mathrm{C}[0]\end{array}$ & $\begin{array}{c}\text { ArtGallery } \\
\mathrm{M}[1.0]\end{array}$ & $\begin{array}{c}\text { Shopping Center } \\
\text { R [0] }\end{array}$ & 0.35 \\
\hline$o f v_{16}$ & $\begin{array}{l}\text { Pension } \\
\text { S [0.38] } \\
\end{array}$ & $\begin{array}{l}\text { LightMeal } \\
\text { R [0] }\end{array}$ & $\begin{array}{c}\text { ArcheologicalSite } \\
\text { R [0.08] }\end{array}$ & $\begin{array}{c}\text { CarRental } \\
{[0]}\end{array}$ & 0.29 \\
\hline
\end{tabular}

Note that in the case of a feature associated with a weight equal to zero, such as for instance InternationalMeal (see the ontology in Figure 1), we assume that consim between this feature and any other feature is equal to zero as well. This is the reason why in Table 5, in the case of the of $v_{7}$, the pair RegularMeal, InternationalMeal has a consim value equal to zero, even though they are in a parent/child relation (and the same results are obtained for the other features in the InternationalMeal column with different kinds of relations).

Table 6. Ranking results for $r v_{3}$

\begin{tabular}{|c|c|c|c|c|c|c|}
\hline$r v_{3}$ & Pension & MediterraneanMeal & Cinema & ShoppingCenter & & Semsim \\
\hline$o f v_{11}$ & $\begin{array}{l}\text { RegularAcc } \\
\mathrm{H}(1)[0.49]\end{array}$ & $\begin{array}{l}\text { LightMeal } \\
\mathrm{H}(1)[0.32]\end{array}$ & $\begin{array}{l}\text { Cinema } \\
\mathrm{M}[1.0]\end{array}$ & $\begin{array}{l}\text { Bazaar } \\
\mathrm{S} \text { [0.62] }\end{array}$ & & 0.61 \\
\hline$o f v_{2}$ & $\begin{array}{l}\text { Pension } \\
\mathrm{M}[1.0]\end{array}$ & $\begin{array}{c}\text { VegetarianMeal } \\
\mathrm{S}[0.23] \\
\end{array}$ & $\begin{array}{c}\text { ArtGallery } \\
\text { C [0.07] }\end{array}$ & $\begin{array}{c}\text { ShoppingCenter } \\
\text { M [1.0] } \\
\end{array}$ & & 0.58 \\
\hline$o f v_{1}$ & $\begin{array}{c}\text { InternationalHotel } \\
\mathrm{S}[0.39] \\
\end{array}$ & $\begin{array}{c}\text { FrenchMeal } \\
\text { C [0.02] } \\
\end{array}$ & $\begin{array}{l}\text { Cinema } \\
\mathrm{M}[1.0]\end{array}$ & $\begin{array}{c}\text { Flight } \\
\mathrm{R}[0] \\
\end{array}$ & & 0.35 \\
\hline$o f v_{14}$ & $\begin{array}{l}\text { RegularAcc } \\
\mathrm{H}(1)[0.49] \\
\end{array}$ & $\begin{array}{l}\text { RegularMeal } \\
\text { R [0.02] }\end{array}$ & $\begin{array}{l}\text { Museum } \\
\mathrm{R}[0.10]\end{array}$ & $\begin{array}{c}\text { Bazaar } \\
\mathrm{S}[0.62] \\
\end{array}$ & & 0.31 \\
\hline$o f v_{6}$ & $\begin{array}{c}\text { SeasideCottage } \\
\mathrm{C}[0] \\
\end{array}$ & $\begin{array}{c}\text { LightMeal } \\
\mathrm{H}(1)[0.32] \\
\end{array}$ & $\begin{array}{c}\text { ArcheologicalSite } \\
\mathrm{R}[0.10]\end{array}$ & $\begin{array}{c}\text { ShoppingCenter } \\
\text { M [1.0] } \\
\end{array}$ & $\begin{array}{c}\text { Flight } \\
{[0]}\end{array}$ & 0.28 \\
\hline$o f v_{16}$ & $\begin{array}{l}\text { Pension } \\
\mathrm{M}[1.0] \\
\end{array}$ & $\begin{array}{c}\text { LightMeal } \\
\mathrm{H}(1)[0.32]\end{array}$ & $\begin{array}{c}\text { ArcheologicalSite } \\
\mathrm{R}[0.10]\end{array}$ & $\begin{array}{c}\text { CarRental } \\
\mathrm{R}[0] \\
\end{array}$ & $\begin{array}{c}\text { Flight } \\
{[0]}\end{array}$ & 0.28 \\
\hline
\end{tabular}

Table 7. Ranking results for $r v_{4}$

\begin{tabular}{|c|c|c|c|c|c|c|}
\hline$r v_{4}$ & CountryResort & LightMeal & Museum & ArcheologicalSite & Train & Semsim \\
\hline$o f v_{18}$ & $\begin{array}{l}\text { CozyAcc } \\
\mathrm{H}(1)[0.70]\end{array}$ & $\begin{array}{c}\text { VegetarianMeal } \\
\mathrm{H}(1)[0.46]\end{array}$ & $\begin{array}{c}\text { Exibition } \\
\mathrm{S}[0.15]\end{array}$ & $\begin{array}{c}\text { ArcheologicalSite } \\
\text { M [1.0] }\end{array}$ & $\begin{array}{c}\text { Train } \\
\mathrm{M}[1.0]\end{array}$ & 0.66 \\
\hline$o f v_{4}$ & $\begin{array}{c}\text { CozyAcc } \\
\mathrm{H}(1)[0.70]\end{array}$ & $\begin{array}{c}\text { VegetarianMeal } \\
\text { H(1) }[0.46]\end{array}$ & $\begin{array}{l}\text { Museum } \\
\mathrm{M}[1.0]\end{array}$ & & $\begin{array}{c}\text { Train } \\
\text { M [1.0] }\end{array}$ & 0.63 \\
\hline$o f v_{6}$ & $\begin{array}{c}\text { SeasideCottage } \\
\mathrm{S}[0.54] \\
\end{array}$ & $\begin{array}{l}\text { LightMeal } \\
\mathrm{M}[1.0]\end{array}$ & $\begin{array}{c}\text { ShoppingCenter } \\
\text { R [0] }\end{array}$ & $\begin{array}{c}\text { ArcheologicalSite } \\
\text { M [1.0] }\end{array}$ & $\begin{array}{c}\text { Flight } \\
\mathrm{S}[0.18]\end{array}$ & 0.55 \\
\hline$o f v_{16}$ & $\begin{array}{c}\text { Pension } \\
\mathrm{C}[0] \\
\end{array}$ & $\begin{array}{l}\text { LightMeal } \\
\text { M [1.0] }\end{array}$ & $\begin{array}{c}\text { CarRental } \\
\mathrm{C}[0]\end{array}$ & $\begin{array}{c}\text { ArcheologicalSite } \\
\text { M [1.0] }\end{array}$ & $\begin{array}{c}\text { Flight } \\
\mathrm{S}[0.18] \\
\end{array}$ & 0.44 \\
\hline$o f v_{22}$ & $\begin{array}{l}\text { FarmHouse } \\
\quad \mathrm{C}[0]\end{array}$ & $\begin{array}{l}\text { LightMeal } \\
\text { M [1.0] }\end{array}$ & & $\begin{array}{c}\text { RockConcert } \\
\text { R [0.11] }\end{array}$ & $\begin{array}{c}\text { Train } \\
\mathrm{M}[1.0]\end{array}$ & 0.42 \\
\hline
\end{tabular}


Note that in some cases the ofvs may have high SemSim values even though in the presence of dangling features of the request vector. This is the case, for instance, of the of $v_{15}$ in Table 5, which is associated with the highest SemSim value in the list (0.66). In fact, it presents two exact matches (InternationalHotel) and (Flight), one sibling relation (ArtGallery, PicureGallery), and the InternationalMeal feature of the request vector $r v_{2}$ that is dangling.

\section{$5 \quad$ Visualization Methods for Results}

In order to provide a better visualization of the results of the SemSim method, we show them in graphical ways. In particular, we adopt radial and radar representations. The former gives a general idea of the results, while the latter focuses on a particular $o f v$. In the radial representation, given a request vector (rv) we place the SemSim values for the different $o f v$ s on concentric circumferences where the radius of each circumference corresponds to the SemSim value of the related ofv. In Figure 2, an example related to the $r v_{4}$ is given, where the first five ofvs in the ranking have been reported according to Table 7 .

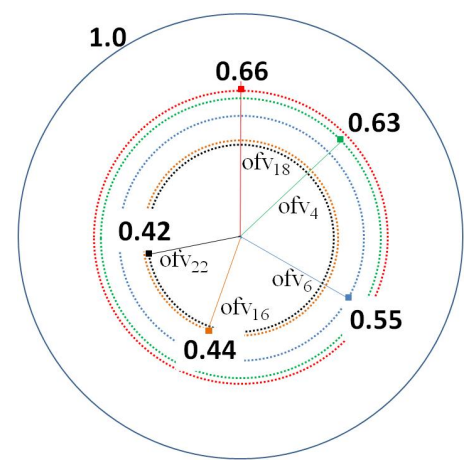

Fig. 2. Radial representation for $r v_{4}$

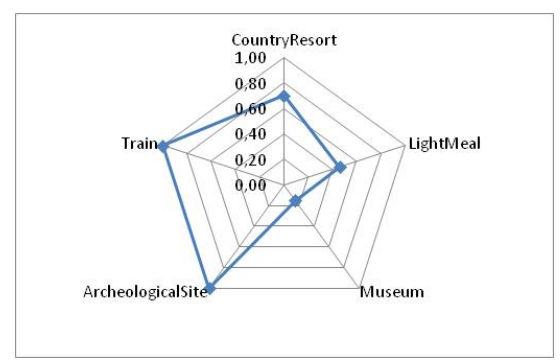

Fig. 3. Radar representation $\left(o f v_{18}, r v_{4}\right)$

In the radar representation, given a $r v$ and an ofv, we show all the features of the $r v$, and for each of these features, the consim value obtained from the pairs formed with the concepts of the $o f v$, as shown in Table 7. For instance, $r v_{4}$ is composed by five features: CountryResort, LightMeal, Museum, ArcheologicalSite, and Train. Therefore, $r v_{4}$ is represented as a pentagon, where each vertex is labeled with one of its features. As illustrated in Figure 3, the radar representation shows how the $r v_{4}$ is approximated by the of $v_{18}$.

\section{Conclusions}

In this paper, we have addressed SemSim, a method for the semantic search and retrieval of resources that populate a search space. SemSim, which has been already 
experimented in the tourism sector, has been further investigated. In particular, a qualitative analysis has been given by providing the user with an explanation about the mechanism adopted in deriving the ranked list of objects. Furthermore, graphical representations about the results have also been given.

As a future work, we plan to study ontology comprising different types of ontological relations.

\section{References}

1. Batzios, A., Mitkas, P.A.: WebOWL: A Semantic Web search engine development experiment. Expert Systems with Applications (ESWA 2012) 39(5), 5052-5060 (2012)

2. Castano, S., De Antonellis, V., Fugini, M.G., Pernici, B.: Conceptual Schema Analysis: Techniques and Applications. ACM Transactions on Databases Systems 23(3), 286-333 (1998)

3. Euzenat, J., Shvaiko, P.: Ontology Matching. Springer (2007)

4. Fang, W.-D., Zhang, L., Wang, Y.-X., Dong, S.-B.: Towards a Semantic Search Engine Based on Ontologies. In: Proceedings of 4th International Conference on Machine Learning, Guangzhou (2005)

5. Formica, A., Missikoff, M., Pourabbas, E., Taglino, F.: Semantic search for matching user requests with profiled enterprises. Computers in Industry 64, 191-202 (2013)

6. Francis, W.N., Kucera, H.: Brown Corpus Manual. Providence, Rhode Island. Department of Linguistics, Brown University (1979)

7. Gruber, T.R.: A translation approach to portable ontologies. Knowledge Acquisition 5(2), 199-220 (1993)

8. Kim, J.W., Candan, K.S.: CP/CV: Concept Similarity Mining without Frequency Information from Domain Describing Taxonomies. In: Proceedings of Conference on Information and Knowledge Management, CIKM 2006 (2006)

9. Kuhn, H.W.: Variants of the Hungarian method for assignment problems. Naval Research Logistics Quarterly 3, 253-258 (1956)

10. Lee, M., Kim, W., Park, S.: Searching and ranking method of relevant resources by user intention on the Semantic Web. Expert Systems with Applications (ESWA 2012) 39(4), 4111-4121 (2012)

11. Lin, D.: An Information-Theoretic Definition of Similarity. In: Shavlik, J.W. (ed.) Proceedings of 15th the International Conference on Machine Learning, pp. 296-304. Morgan Kaufmann, Madison (1998)

12. Maarek, Y.S., Berry, D.M., Kaiser, G.E.: An Information Retrieval Approach For Automatically Constructing Software Libraries. IEEE Transactions on Software Engineering 17(8), 800-813 (1991)

13. Maguitman, A.G., Menczer, F., Roinestad, H., Vespignani, A.: Algorithmic Detection of Semantic Similarity. In: Proceedings of World Wide Web Conference (WWW 2005) Conference, Chiba, Japan (May 2005)

14. Mairson, H.: The Stable Marriage Problem. The Brandeis Review 12(1) (1992)

15. Melo, D., Pimenta Rodrigues, I., Beires Nogueira, V.: Work Out the Semantic Web Search: The Cooperative Way. In: Advances in Artificial Intelligence (ADVAI 2012) (2012)

16. Miller, G.A.: Wordnet: A Lexical database for English. Communication of the ACM 38(11), 39-41 (1995) 